\title{
A piecewise partitioning Scaled Boundary Finite Element algorithm to solve viscoelastic problems with cyclic symmetry
}

\author{
Chongshuai Wang, Yiqian He, Haitian Yang* \\ State Key Lab of Structural Analysis for Industrial Equipment \\ Dept. of Engineering Mechanics \\ Dalian University of Technology, Dalian, 116024 \\ Dalian, P. R. China \\ September, 2016 \\ *Email: haitian@dlut.edu.cn
}

\begin{abstract}
Scaled Boundary Finite Element Method (SBFEM) and a temporally adaptive algorithm are combined to solve viscoelastic problems. By expanding variables at a discretized time interval, a spatially and temporally coupled viscoelastic problem is decoupled into a series of recursive spatial problems, which are solved by SBFEM, the computing accuracy in the time domain is controlled via a self-adaptive process. For the cyclic symmetric structures, the cyclic symmetry is exploited to reduce the computational expense of SBFEM, both the eigenvalue and system equations of SBFEM are partitioned into a number of smaller independent problems, which are solved by a partitioning algorithm. Two numerical examples are given to verify and illustrate the proposed approach.
\end{abstract}

Key Words: Viscoelasticity, Cyclic symmetry, SBFEM, Computational expense.

\section{Introduction}

The numerical analysis for viscoelastic materials/structures is an important issue in many aspects of engineering [1], particularly because the analytical solution is limited due to the time dependent constitutive relationship, the complex geometry and boundary conditions. A numerical approach of viscoelastic analysis mainly consists of two parts. One is related to spatial discretized algorithm such as FEM [2] and [3], EFG [4] and [5], BEM [6], etc, and another is associated with the descritized algorithm in the time domain, such as various kinds of FDM [7] and [8].

The Scaled Boundary Finite Element Method (SBFEM) [9] and [10] is a spatial discretized algorithm that is semi-analytical, and exhibits good performence in dealing 
with problems of stress singularities and unbounded domains [11], [12], [13], [14], [15], [16], [17] and [18]

On the other hand, there are numbers of discretized algorithms in the time domain employed to solve viscoelastic problems, among which a temporally-piecewise algorithm [19], [20] and [21] is one that is not only easy to combine with spatially discretized algorithms, but also able to adapt the change of the size of time step via a self-adaptive process.

This paper attempts to combine advantages of SBFEM and the temporally-piecewise adaptive algorithm, and proposes a new approach to solve viscoelastic problems.

It is noteworthy that SBFEM is more computationally expensive than FEM in an elastic analysis mainly because an eigenvalue problem needs to be solved in generating system equations [22]. If SBFEM is emplyed at every discritized time interval in the numerical analysis for the viscoelastic problem [23], the computational cost will be more expensive. Therefore, a piecewise partitioning algorithm [22] is proposed for a special kind of structure, i.e. structure with cyclic symmetry that can be observed in many engineering equipment and structures, such as aircraft rotor, turbine blades, satellite antenna dishes, and some large space structures etc [5] and [22]. A proof that the recursive eigenvalue matrices of SBFEM equations are block-circulant is presented, and on the basis of this proof, both the recursive eigenvalue and system equations of SBFEM are partitioned into a number of smaller independent problems, which are solved by a partitioning algorithm.

The benefits gained using the SBFEM based piecewise partitioning algorithm are two folds. (1) In the spacial domain, the implementation of SBFEM may further improve computing accuracy, particularly for the problems of stress singularities and unbounded boundary, the computational cost will be reduced by solving a series of independent subproblems instead of the whole SBFEM eigenvalue and system equations. (2) In the time domain, variables can be described more precisely, and computing accuracy can be controlled via an adaptive process.

The paper commences with the recursive govening equations for the viscoelastic problem in the section 2; section 3 derives recursive SBFEM equations; section 4 sets up recursive partioning SBFEM equations; section 5 provides a numerical verification via two examples, and section 6 gives conclusions. 


\section{Recursive governing equations for viscoelastic problems}

The governing equations of viscoelasticity can be described by [24]

$$
\begin{aligned}
& {[H]\{\sigma\}+\{b\}=\{O\}} \\
& \{\varepsilon\}=[H]^{T}\{u\}
\end{aligned}
$$

where

$$
[H]=\left[\begin{array}{ccc}
\partial / \partial x & 0 & \partial / \partial y \\
0 & \partial / \partial y & \partial / \partial x
\end{array}\right]
$$

The boundary conditions are specified by [24]

$$
\begin{array}{ll}
\{u\}=\{\tilde{u}\} & \text { on } \Gamma_{u} \\
\{p\}=\{\tilde{p}\} & \text { on } \Gamma_{\sigma}
\end{array}
$$

where $\{\sigma\}$ and $\{\varepsilon\}$ denote the vector of stress and strain, respectively, $\{b\}$ is the vector of body force, $\{u\}$ is the vector of displacement, $\{p\}$ denotes the vector of traction, $\{\tilde{u}\},\{\tilde{p}\}$ are the prescribed values of $\{u\}$ and $\{p\}$ on the boundary, respectively. $\Gamma=\Gamma_{u}+\Gamma_{\sigma}$ represents the boundary of $\Omega$, and the subscript $u$ and $\sigma$ refer to stress and displacement, respectively.

We divide time domain into a number of intervals where the initial points and the sizes of time intervals are defined by $t_{0}, t_{1}, t_{2}, \ldots, t_{k}, \ldots$ and $\Delta t_{0}, \Delta t_{1}, \Delta t_{2}, \ldots, \Delta t_{k}, \ldots$, respectively. At a discretized time interval, in order to describe the variation of variables more precisely, all variables are expanded in the term of $s$

$$
\begin{gathered}
\{\sigma\}=\sum_{m=0}\left\{\sigma_{m}\right\} s^{m} \\
\{\varepsilon\}=\sum_{m=0}\left\{\varepsilon_{m}\right\} s^{m} \\
\{b\}=\sum_{m=0}\left\{b_{m}\right\} s^{m} \\
\{u\}=\sum_{m=0}\left\{u_{m}\right\} s^{m} \\
\{\tilde{u}\}=\sum_{m=0}\left\{\tilde{u}_{m}\right\} s^{m} \\
\{p\}=\sum_{m=0}\left\{p_{m}\right\} s^{m} \\
\{\tilde{p}\}=\sum_{m=0}\left\{\tilde{p}_{m}\right\} s^{m}
\end{gathered}
$$




$$
s=\frac{t-t_{k-1}}{\Delta t_{k}}
$$

where $t_{k-1}$ and $\Delta t_{k}$ represent the initial point and size of the $k$-th time interval, respectively. $\left\{\sigma_{m}\right\}$ and $\left\{\varepsilon_{m}\right\}$ denote the expanding coefficients of $\{\sigma\}$ and $\{\varepsilon\}$, respectively, $\left\{b_{m}\right\},\left\{u_{m}\right\},\left\{p_{m}\right\},\left\{\tilde{u}_{m}\right\}$ and $\left\{\tilde{p}_{m}\right\}$ denote the expanding coefficients of $\{b\},\{u\},\{p\},\{\tilde{u}\}$ and $\{\tilde{p}\}$, respectively.

In this paper, we assume $\Delta t_{k}=\Delta t, k=0,1,2 \ldots$

The conversion relationship of differential from $t$ to $s$ is

$$
\frac{d}{d t}=\frac{1}{\Delta t} \frac{d}{d s} ; \quad \frac{d^{2}}{d t^{2}}=\frac{1}{\Delta t^{2}} \frac{d^{2}}{d s^{2}}
$$

Substituting Eqs. (6-12) into Eqs. (1-5) then yields

$$
\begin{array}{ll}
{[H]\left\{\sigma_{m}\right\}+\left\{b_{m}\right\}=\{0\}} & \\
\left\{\varepsilon_{m}\right\}=[H]^{T}\left\{u_{m}\right\} & \\
\left\{u_{m}\right\}=\left\{\tilde{u}_{m}\right\} & \text { on } \Gamma_{u} \\
\left\{p_{m}\right\}=\left\{\tilde{p}_{m}\right\} & \text { on } \Gamma_{\sigma}
\end{array}
$$

For the three-parameter solid viscoelastic model (Fig. 1) [25], the constitutive relationship can be written in a differential form

$$
\begin{aligned}
& q_{0}\{\varepsilon(t)\}+q_{1} \frac{d\{\varepsilon(t)\}}{d t}=[G]\left(\{\sigma(t)\}+p_{1} \frac{d\{\sigma(t)\}}{d t}\right) \quad(t>0) \\
& \{\varepsilon(t)\}=\frac{1}{E_{2}}[G]\{\sigma(t)\} \quad(t=0)
\end{aligned}
$$

where

$$
\begin{aligned}
& p_{1}=\frac{\eta_{1}}{E_{1}+E_{2}} ; q_{0}=\frac{E_{1} E_{2}}{E_{1}+E_{2}} ; q_{1}=\frac{E_{2} \eta_{1}}{E_{1}+E_{2}} \\
& {[G]=\left[\begin{array}{ccc}
G_{11} & G_{12} & 0 \\
& G_{22} & 0 \\
\text { symm } & & G_{33}
\end{array}\right]}
\end{aligned}
$$

For the plane stress problem

$$
G_{11}=G_{22}=1 ; G_{33}=2(1+v) ; G_{12}=-v
$$

For the plane strain problem 


$$
G_{11}=G_{22}=1-v^{2} ; G_{33}=2(1+v) ; G_{12}=-v(1+v)
$$

where $v, E_{1}, E_{2}$ and $\eta_{1}$ represent constitutive parameters.

Substituting Eqs. (6), (7) and Eq. (14) into Eq. (19) and equating the power of the two sides of the equation then gives

$$
\left\{\sigma_{m}\right\}=[D]\left\{\varepsilon_{m}\right\}+\left\{C_{m}\right\}, \quad m=1,2,3, \ldots \ldots
$$

where

$$
\begin{aligned}
\left\{C_{m}\right\} & =\frac{\Delta t}{m}\left(\frac{1}{\eta_{1}}[D]\left\{\varepsilon_{m-1}\right\}-\frac{1}{p_{1}}\left\{\sigma_{m-1}\right\}\right) \\
{[D] } & =\left(\begin{array}{ccc}
D_{11} & D_{12} & 0 \\
& D_{22} & 0 \\
\text { symm } & & D_{33}
\end{array}\right)
\end{aligned}
$$

For the plane stress problem

$$
D_{11}=s_{1}+\frac{v}{s_{2}} ; \quad D_{22}=\frac{1}{s_{2}} ; \quad D_{33}=\frac{1}{s_{3}} ; \quad D_{12}=\frac{v}{s_{2}}
$$

where

$$
s_{1}=\frac{q_{1}}{p_{1}} ; \quad s_{2}=\left(1-v^{2}\right) \frac{p_{1}}{q_{1}} ; \quad s_{3}=2(1+v) \frac{p_{1}}{q_{1}}
$$

For the plane strain problem; $E_{1}, E_{2}$ and $v$ need to be replaced by $\frac{E_{1}}{1-v^{2}}$, $\frac{E_{2}}{1-v^{2}}$ and $\frac{v}{1-v^{2}}$, respectively.

At the first time inverval.

$$
\left\{\sigma_{0}\right\}=[D]\left\{\varepsilon_{0}\right\}
$$

At the $(k+1)$ th time interval

$$
\left\{\begin{array}{l}
\left\{\varepsilon_{0}\right\}_{(k+1)}=\sum_{i=0}\left\{\varepsilon_{i}\right\}_{k} \\
\left\{\sigma_{0}\right\}_{(k+1)}=\sum_{i=0}\left\{\sigma_{i}\right\}_{k}
\end{array}, \quad k=1,2,3, \ldots,\right.
$$

where subscript $(k+1)$ and $k$ refer to $(k+1)$ th and $k$ th time intervals, respectively.

\section{Recursive SBFEM equations}

The Scaled Boundary Finite Element Method (SBFEM) [9], [10] and [11] introduces a normalised radial coordinate system by scaling a defining curve (usually the domain 
boundary or a part of the domain boundary) relative to a scaling centre $\left(x_{0}, y_{0}\right)$ selected within the domain or at the intersection of two straight sections of the boundary (Fig.2). The normalised radial coordinate $\xi$ runs from the scaling centre towards the defining curve, and has values of zero at the scaling centre and unity at the defining curve. The other circumferential coordinate $\eta$ specifies a distance around the defining curve from an origin on the curve. The scaled boundary and Cartesian coordinate systems are related by the scaling equations

$$
\begin{aligned}
& x=x_{0}+\xi x_{\eta}(\eta) \\
& y=y_{0}+\xi y_{\eta}(\eta)
\end{aligned}
$$

Displacement and stress components are retained in the original Cartesian coordinate directions, while position is specified in terms of the scaled boundary coordinates.

For the problem defined by Eqs. (15-18), an approximate solution of $\left\{u_{m}(\xi, \eta)\right\}$ is sought in the form [26]

$$
\left\{u_{m}(\xi, \eta)\right\}=[N(\eta)]\left\{u_{m}(\xi)\right\}
$$

where $[N(\eta)]$ is a matrix of shape functions.

By the virtue of the weighted residual method [11], one has (in the absence of body force)

$$
\int_{V}\{\delta \varepsilon(\xi, \eta)\}^{T}\{\sigma(\xi, \eta, t)\} \mathrm{d} V=\int_{\eta}\{\delta u(\eta)\}^{T}\{p(\eta, t)\} \mathrm{d} \eta
$$

Substituting Eq. (6) into Eq. (35) then yields

$$
\int_{V}\{\delta \varepsilon(\xi, \eta)\}^{T}\left\{\sigma_{m}(\xi, \eta)\right\} \mathrm{d} V=\int_{S}\{\delta u(\eta)\}^{T}\left\{p_{m}(\eta)\right\} \mathrm{ds}
$$

Substituting Eq. (25) into Eq. (36) gives

$$
\int_{V}\{\delta \varepsilon(\xi, \eta)\}^{T}[D]\left\{\varepsilon_{m}(\xi, \eta)\right\} \mathrm{d} V=\int_{\eta}\{\delta u(\eta)\}^{T}\left\{p_{m}(\eta)\right\} \mathrm{ds}-\int_{V}\{\delta \varepsilon(\xi, \eta)\}^{T}\left\{C_{m}\right\} \mathrm{d} V
$$

The left-side of Eq. (37) becomes

$$
\begin{aligned}
\int_{V}\{\delta \varepsilon(\xi, \eta)\}^{T}[D]\left\{\varepsilon_{m}(\xi, \eta)\right\} \mathrm{d} V \\
=\{\delta u\}^{T}\left\{\left[E^{0}\right]\left\{u_{m}\right\}_{, \xi}+\left[E^{1}\right]\left\{u_{m}\right\}\right\} \\
-\int_{0}^{1}\{\delta u(\xi)\}^{T}\left\{\left[E^{0}\right] \xi\left\{u_{m}(\xi)\right\}_{, \xi \xi}+\left[\left[E^{0}\right]+\left[E^{1}\right]^{T}-\left[E^{1}\right]\right]\left\{u_{m}(\xi)\right\}_{, \xi}-\left[E^{2}\right] \frac{1}{\xi}\left\{u_{m}(\xi)\right\}\right\} \mathrm{d} \xi
\end{aligned}
$$


where

$$
\begin{aligned}
& {\left[E^{0}\right]=\int_{\eta}\left[B^{1}(\eta)\right]^{T}[D]\left[B^{1}(\eta)\right]|J| \mathrm{d} \eta} \\
& {\left[E^{1}\right]=\int_{\eta}\left[B^{2}(\eta)\right]^{T}[D]\left[B^{1}(\eta)\right]|J| \mathrm{d} \eta} \\
& {\left[E^{2}\right]=\int_{\eta}\left[B^{2}(\eta)\right]^{T}[D]\left[B^{2}(\eta)\right]|J| \mathrm{d} \eta}
\end{aligned}
$$

The right-side of Eq. (37) is written as

$$
\{\delta u\}^{T}\left\{P_{m}\right\}=\{\delta u\}^{T}\left(\left\{P S_{m}\right\}-\left\{P C_{m}\right\}\right)
$$

where

$$
\begin{aligned}
& \left\{P S_{m}\right\}=\int_{\eta}[N(\eta)]\left\{p_{m}(\eta)\right\} \mathrm{d} \eta \\
& \{\delta u\}^{T}\left\{P C_{m}\right\}=\int_{V}\{\delta \varepsilon(\xi, \eta)\}^{T}\left\{C_{m}\right\} \mathrm{d} V
\end{aligned}
$$

The arbitrariness of $\{\delta u(\xi)\}$ leads to

$$
\begin{aligned}
& \left\{P_{m}\right\}=\left[E^{0}\right]\left\{u_{m}\right\}_{, \xi}+\left[E^{1}\right]^{T}\left\{u_{m}\right\} \\
& {\left[E^{0}\right] \xi^{2}\left\{u_{m}(\xi)\right\}_{, \xi \xi}+\left[\left[E^{0}\right]+\left[E^{1}\right]^{T}-\left[E^{1}\right]\right] \xi\left\{u_{m}(\xi)\right\}_{, \xi}-\left[E^{2}\right]\left\{u_{m}(\xi)\right\}=\{0\}}
\end{aligned}
$$

By inspection, solutions to the homogeneous set of Euler-Cauchy differential equations represented by Eq. (46) must be of the form [11]

$$
\left\{u_{m}(\xi)\right\}=c_{1} \xi^{-\lambda_{1}}\left\{\phi_{1}\right\}+c_{2} \xi^{-\lambda_{2}}\left\{\phi_{2}\right\}+\ldots
$$

$\left\{\phi_{i}\right\}$ and $\lambda_{i}$ are obtained by solving the following eigenproblem [11]

$$
\left[\begin{array}{cc}
{\left[E^{0}\right]^{-1}\left[E^{1}\right]^{T}} & -\left[E^{0}\right]^{-1} \\
{\left[E^{1}\right]\left[E^{0}\right]^{-1}\left[E^{1}\right]^{T}-\left[E^{2}\right]} & -\left[E^{1}\right]\left[E^{0}\right]^{-1}
\end{array}\right]\left\{\begin{array}{l}
\{\phi\} \\
\{q\}
\end{array}\right\}=\lambda\left\{\begin{array}{l}
\{\phi\} \\
\{q\}
\end{array}\right\}
$$

At $\xi=1$

$$
\{c\}=[\Phi]^{-1}\left\{u_{m}\right\}
$$

where

$$
[\Phi]=\left[\left\{\phi_{1}\right\},\left\{\phi_{2}\right\}, \ldots . .\left\{\phi_{n}\right\}\right]
$$

Furthermore

$$
\left\{u_{m}\right\}=[K]^{-1}\left\{P_{m}\right\}
$$

where 


$$
\begin{aligned}
& {[K]=[Q][\Phi]^{-1}} \\
& {[\mathrm{Q}]=\left[\left\{q_{1}\right\},\left\{q_{2}\right\}, \ldots . .\left\{q_{n}\right\}\right]} \\
& \left\{P_{m}\right\}=\left\{P S_{m}\right\}-\left\{P C_{m}\right\} \\
& \left\{P C_{m}\right\}=\frac{\Delta t}{m}\left(\left(\frac{1}{\eta_{1}}-\frac{1}{p_{1}}\right)[K]\left\{u_{m-1}\right\}-\frac{1}{p_{1}}\left\{P C_{m-1}\right\}\right)
\end{aligned}
$$

$\left\{q_{i}\right\}$ refers to the dual variable of $\left\{\phi_{i}\right\}[11]$.

At the initial points of $k$ th time interval, $\left\{u_{0}\right\}$ is determined by

$$
\begin{array}{ll}
\left\{u_{0}\right\}^{k}=[K]^{-1}\left\{P S_{0}\right\} ; & \text { if } k=1 \\
\left\{u_{0}\right\}^{k}=\sum_{m}\left\{u_{m}\right\}^{k-1} & \text { if } k>1
\end{array}
$$

An adaptive computing process at a time interval can be realized with the increase of $m$ until the following criteria is satisfied

$$
\frac{\left\|\left.\{u\}^{m} s^{m}\right|_{s=1}\right\|_{2}}{\left\|\left.\sum_{j=0}^{m-1}\{u\}^{m} s^{m}\right|_{s=1}\right\|_{2}} \leq \beta
$$

where $\beta$ is a prescribed error tolerance, and $\|\bullet\|_{2}$ represents a $\mathrm{L}^{2}$-norm.

\section{Recursive partitioning algorithm}

A structure or a computational region $\Omega$ is said to possess cyclic symmetry of order $N$ when its geometry and physical properties are invariant under the following $N$ symmetry transformations [4]

$$
\Psi_{i}=(i-1) \theta, \quad i=1,2, . N .
$$

where $\Psi_{i}$ represents a rotation of $\Omega$ about its axis of rotation with an angle of $\theta=2 \pi / N$, and $N$ is defined as the order of symmetry. For example, Fig. 3 shows a cyclic symmetric plate with $N=6$.

To make full use of the symmetry in SBFE analysis, nodes are required to be arranged in a symmetric way so that original symmetry of the system is retained.

$\Omega$ is naturally divided into $N$ identical parts $\Omega_{i}, i=1,2, \ldots, N$.

$$
\Omega=\Omega_{1} \cup \Omega_{2} \cdots \cup \Omega_{N}, \Omega_{i}=\Psi_{i}: \Omega_{1}
$$

$\Omega_{i}$ is arranged in an anti-clock sequence 
Eq. (59) means that $\Omega_{i}$ can be obtained from $\Omega_{1}$, which is called the 'basic region' and can be arbitrarily chosen from those identical parts. By setting up nodal co-ordinates and integration points on $\Omega_{1}$ only, one can then obtain the complete computational model by using Eq. (59). For any node or integration point in the basic region, there are another $N-1$ different nodes or integration points, which are located symmetrically on the other $N-1$ symmetry regions. These $N$ nodes constitute a set of symmetric nodes, which is called a symmetric node orbit, and is designated as $O_{A}$

$$
O_{A}=\left\{A_{1}, A_{2}, \ldots, A_{N}\right\}
$$

For the $N$ nodes of $O_{A}$, the reference co-ordinate directions of node $A_{1}$, which belongs to the basic region, are first defined, and then the reference co-ordinate directions for the other symmetric nodes can be defined by using Eq. (59) (see Fig.3, for example.) It is readily seen that the six interface nodes $B_{i}(i=1,2, \ldots, N)$ constitute orbit $O_{B}$. Only those nodes that are located on the internal part and the 'right' interface of $\Omega_{1}$ are regarded as belonging to the basic region. For example, of the two interface nodes of the basic region in Fig. 3, only $B_{1}$ is regarded as belonging to $\Omega_{1}$, while $B_{2}$ is regarded as belonging to $\Omega_{2}$. Assuming the number of nodes belonging to $\Omega_{1}$ is $d$, the total number is $N \cdot d$.

For a viscoelastic cyclic symmetric system with order $N,\{u\}$ is designated by [3]

$$
\{u\}^{T}=\left(\{u\}^{1 T},\{u\}^{2 T}, \ldots,\{u\}^{N T}\right)
$$

where the nodal vector $\{u\}^{i}$ belonging to the $i$ th symmetric region is a sub-vector of $\{u\}$ with dimension $2 d$.

Therefore

$$
\left\{u_{m}\right\}^{T}=\left(\left\{u_{m}\right\}^{1 T},\left\{u_{m}\right\}^{2 T}, \ldots,\left\{u_{m}\right\}^{N T}\right)
$$

Using a symmetry-adapted reference coordinate system [22], the vector $\left\{u_{m}\right\}$, $\left\{P_{m}\right\},\{\varphi\}$ and $\{q\}$ are expressed via

$$
\begin{aligned}
& \left\{u_{m}\right\}=[T]^{g}\left\{\overline{u_{m}}\right\} \\
& \left\{P_{m}\right\}=[T]^{g}\left\{\overline{P_{m}}\right\}=[T]^{g}\left(\left\{\overline{P S_{m}}\right\}-\left\{\overline{P C_{m}}\right\}\right) \\
& \{\varphi\}=[T]^{g}\{\bar{\varphi}\} \\
& \{q\}=[T]^{g}\{\bar{q}\}
\end{aligned}
$$




$$
\{q\}=[T]^{g}\{\bar{q}\}
$$

where the overbar refers to vectors defined in the symmetry-adapted system [4]

$$
\begin{aligned}
& {[T]^{g}=\left[\begin{array}{cccc}
{[\bar{T}]_{1}^{g}} & & & 0 \\
& {[\bar{T}]_{2}^{g}} & & \\
& & \cdots & \\
0 & & & {[\bar{T}]_{N}^{g}}
\end{array}\right]_{n \times n}} \\
& {[\bar{T}]_{i}^{g}=\left[\begin{array}{cccc}
{[T]_{i}} & & & 0 \\
& {[T]_{i}} & & \\
& & \ldots & \\
0 & & & {[T]_{i}}
\end{array}\right]_{2 d \times 2 d},} \\
& {[T]_{i}=\left[\begin{array}{cc}
\cos (i-1) \theta & -\sin (i-1) \theta \\
\sin (i-1) \theta & \cos (i-1) \theta
\end{array}\right], \quad \theta=2 \pi / N} \\
& {[T]_{i}^{T}[T]_{i}=[I]} \\
& {[T]_{i+k-1}[T]_{i}=[T]_{i}[T]_{k}}
\end{aligned}
$$

Substituting Eqs. (65) and (66) into Eq (48) then yields

$$
\left[\begin{array}{cc}
{[E]_{0}^{-1}[E]_{1}^{T}} & -[E]_{0}^{-1} \\
{[E]_{1}[E]_{0}^{-1}[E]_{1}^{T}-[E]_{2}} & -[E]_{1}[E]_{0}^{-1}
\end{array}\right]\left[\begin{array}{cc}
{[T]^{g}} & 0 \\
0 & {[T]^{g}}
\end{array}\right]\left\{\begin{array}{c}
\{\bar{\varphi}\} \\
\bar{q}\}
\end{array}\right\}=\lambda\left[\begin{array}{cc}
{[T]^{g}} & 0 \\
0 & {[T]^{g}}
\end{array}\right]\left\{\begin{array}{c}
\{\bar{\varphi}\} \\
\bar{q}\}
\end{array}\right\}
$$

Multiplying each side of Eq. (72) by $\left[\begin{array}{cc}{[T]^{g T}} & 0 \\ 0 & {[T]^{g T}}\end{array}\right]$ and applying the identity stated in Eq. (70) to the right hand side then gives

$$
\left[\begin{array}{cc}
{[T]^{g T}} & 0 \\
0 & {[T]^{g T}}
\end{array}\right]\left[\begin{array}{cc}
{[E]_{0}^{-1}[E]_{1}^{T}} & -[E]_{0}^{-1} \\
{[E]_{1}[E]_{0}^{-1}[E]_{1}^{T}-[E]_{2}} & -[E]_{1}[E]_{0}^{-1}
\end{array}\right]\left[\begin{array}{cc}
{[T]^{g}} & 0 \\
0 & {[T]^{g}}
\end{array}\right]\left\{\begin{array}{c}
\{\bar{\varphi}\} \\
\{\bar{q}\}
\end{array}\right\}=\lambda\left\{\begin{array}{c}
\{\bar{\varphi}\} \\
\bar{q}\}
\end{array}\right\}
$$

Eq. (73) can further be written as

$$
\left[\begin{array}{ll}
{[\bar{Z}]_{11}} & {[\bar{Z}]_{12}} \\
{[\bar{Z}]_{21}} & {[\bar{Z}]_{22}}
\end{array}\right]\left\{\begin{array}{c}
\{\bar{\varphi}\} \\
\bar{q}\}
\end{array}\right\}=\lambda\left\{\begin{array}{c}
\{\bar{\varphi}\} \\
\bar{q}\}
\end{array}\right\}
$$

where

$$
\begin{aligned}
& {[\bar{Z}]_{11}=[T]^{g T}[E]_{0}^{-1}[E]_{1}^{T}[T]^{g}} \\
& {[\bar{Z}]_{12}=[T]^{g}[E]_{0}^{1}[T]^{g}} \\
& {[\bar{Z}]_{21}=[T]^{g T}\left([E]_{1}[E]_{0}^{-1}[E]_{1}^{T}-[E]_{2}\right)[T]^{g}} \\
& {[\bar{Z}]_{22}=-[T]^{g T}[E]_{1}[E]_{0}^{-1}[T]^{g}}
\end{aligned}
$$


$[\bar{Z}]_{11},[\bar{Z}]_{12},[\bar{Z}]_{21},[\bar{Z}]_{22}$ can be proved block-circulant [22], having the form

$$
[\bar{Z}]_{i j}=\left[\begin{array}{cccc}
{[\bar{Z}]_{i j}{ }^{1}} & {[\bar{Z}]_{i j}{ }^{2}} & \cdots & {[\bar{Z}]_{i j}{ }^{N}} \\
{[\bar{Z}]_{i j}{ }^{N}} & {[\bar{Z}]_{i j}{ }^{1}} & & \vdots \\
\vdots & & \ddots & {[\bar{Z}]_{i j}{ }^{2}} \\
{[\bar{Z}]_{i j}{ }^{2}} & \cdots & {[\bar{Z}]_{i j}{ }^{N}} & {[\bar{Z}]_{i j}{ }^{1}}
\end{array}\right], \quad i, j=1,2
$$

$\left\{\overline{u_{m}}\right\},\left\{\overline{P_{m}}\right\},\{\bar{\varphi}\}$ and $\{\bar{q}\}$ can further be expressed by [5] and [22]

$$
\begin{aligned}
& \left\{\overline{u_{m}}\right\}=[E]^{g}\left\{\underline{u_{m}}\right\} \\
& \left\{\overline{P_{m}}\right\}=[E]^{g}\left\{\underline{P_{m}}\right\}=[E]^{g}\left(\underline{P S_{m}}-\underline{P C_{m}}\right) \\
& \{\bar{\varphi}\}=[E]^{g}\{\underline{\varphi}\} \\
& \{\bar{q}\}=[E]^{g}\{\underline{q}\}
\end{aligned}
$$

where

$$
[E]^{g}=\left[[e]_{r s s} \cdot[I]^{g}\right]^{T}
$$

$[I]^{g}$ is a unit matrix of $2 d$ dimensions. $[e]_{r s}$ is the ss th element of the basis $[e]_{r}$ defined by

$$
\begin{aligned}
& \{e\}_{1}=\{1,1, \ldots, 1\}^{T} / \sqrt{N} \\
& \{e\}_{2 i}=\sqrt{2 / N}\left\{\cos i \theta_{1}, \cos i \theta_{2}, \ldots, \cos i \theta_{N}\right\}^{T} \\
& \{e\}_{2 i+1}=\sqrt{2 / N}\left\{\sin i \theta_{1}, \sin i \theta_{2}, \ldots, \sin i \theta_{N}\right\}^{T} \\
& \quad i=1, \ldots,[(N-1) / 2], \quad \theta_{k}=(k-1) \theta(k=1,2, \ldots, N) \\
& \quad\{e\}_{N}=\{1,-1,1, \ldots,-1\}^{T} / \sqrt{N} \quad \text { (when } N \text { is even) }
\end{aligned}
$$

where $\left\{\{e\}_{1},\{e\}_{2}, \ldots,\{e\}_{N}\right\}^{T}$ represents a group of complete symmetrized orthogonal unit vectors, $[(N-1) / 2]$ is the largest integer which does not exceed $(N-1) / 2$ [4].

Utilizing Eq. (82) and Eq. (83), Eq. (74) becomes

$$
\left[\begin{array}{ll}
{[\underline{Z}]_{11}} & {[\underline{Z}]_{12}} \\
{[\underline{Z}]_{21}} & {[\underline{Z}]_{22}}
\end{array}\right]\left\{\begin{array}{l}
\{\underline{\varphi}\} \\
\{\underline{q}\}
\end{array}\right\}=\lambda\left\{\begin{array}{l}
\{\underline{\varphi}\} \\
\{\underline{q}\}
\end{array}\right\}
$$

where

$$
\begin{aligned}
& {[\underline{Z}]_{i j}=[E]^{g T}[\bar{Z}]_{i j}[E]^{g}, \quad i, j=1,2} \\
& {[\underline{Z}]_{i j}=\sum_{r=0}^{[N / 2]} \oplus[\underline{Z}]_{i j}{ }_{i j}^{r r} \quad, \quad i, j=1,2}
\end{aligned}
$$


where $\oplus$ represents the direct sum of matrices, i.e., the matrices $[\underline{Z}]_{i j}$ are block-diagonal

$$
\begin{gathered}
{[\underline{Z}]_{i j}{ }^{00}=\left([\bar{Z}]_{i j}{ }^{1}+[\bar{Z}]_{i j}{ }^{2}+\cdots[\bar{Z}]_{i j}{ }^{N}\right)} \\
{[\underline{Z}]_{i j}{ }^{r r}=\left[\begin{array}{ll}
{[\underline{Z}]_{i j}{ }^{r r 11}} & {[\underline{Z}]_{i j}{ }^{r r 12}} \\
{[\underline{Z}]_{i j}{ }^{r r 21}} & {[\underline{Z}]_{i j}{ }^{r r 22}}
\end{array}\right], \quad r=1,2, \ldots,[(N-1) / 2]} \\
{[\underline{Z}]_{i j}{ }^{r r 11}=[\underline{Z}]_{i j}{ }^{r r 22}=\sum_{s=1}^{N}[\bar{Z}]_{i j}{ }^{s} \cos (s s-1) i \theta} \\
{[\underline{Z}]_{i j}{ }^{r r 12}=-[\underline{Z}]_{i j}{ }^{r r 21}=\sum_{s=1}^{N}[\bar{Z}]_{i j}{ }^{s} \sin (s s-1) i \theta} \\
{[\underline{Z}]_{i j}{ }^{N / 2, N / 2}=\left([\bar{Z}]_{i j}{ }^{1}-[\bar{Z}]_{i j}{ }^{2}+[\bar{Z}]_{i j}{ }^{3}-[\bar{Z}]_{i j}{ }^{4} \cdots-[\bar{Z}]_{i j}{ }^{N}\right) \text { when } N \text { is even. }}
\end{gathered}
$$

Eq. (86) is naturally partitioned into $[(N+2) / 2]$ decoupled sub-eigenproblems

$$
\left[\begin{array}{ll}
{[\underline{Z}]_{11}{ }^{r r}} & {[\underline{Z}]_{12}{ }^{r r}} \\
{[\underline{Z}]_{21}{ }^{r r}} & {[\underline{Z}]_{22}{ }^{r r}}
\end{array}\right]\left\{\begin{array}{l}
\{\underline{\varphi}\}^{r} \\
\{\underline{q}\}^{r}
\end{array}\right\}=\lambda\left\{\begin{array}{l}
\{\underline{\varphi}\}^{r} \\
\{\underline{q}\}^{r}
\end{array}\right\}, r=0,1,2, \ldots, N / 2
$$

Therefore, instead of solving the original eigenproblem Eq. (48), now one only needs to solve a series of smaller independent sub-eigenproblems of Eq. (90).

Substituting Eqs. (63), (64), (80) and (81) into Eq. (52) yields

$$
\left\{\underline{u_{m}}\right\}=[\underline{K}]^{-1}\left\{\underline{P_{m}}\right\}
$$

where

$$
\begin{aligned}
&\left\{\underline{\left.P_{m}\right\}}=\right. {[E]^{g T}[T]^{g T}\left\{P_{m}\right\} } \\
&= {[E]^{g T}[T]^{g T}\left\{P S_{m}\right\}+} \\
& \frac{\Delta t}{m}\left(\left(\frac{1}{\eta_{1}}-\frac{1}{p_{1}}\right)[E]^{g T}[T]^{g T}[K]\left\{u_{m-1}\right\}-\frac{1}{p_{1}}[E]^{g T}[T]^{g T}\left\{P C_{m-1}\right\}\right) \\
& {[\underline{K}]=[\underline{Q}][\underline{\Phi}]^{-1} }
\end{aligned}
$$

where $[\underline{\Phi}],[\underline{Q}],\left\{\underline{u_{m}}\right\},\left\{\underline{P_{m}}\right\}$ and $[\underline{K}]$ have the form

$$
[\underline{\Phi}]=\left[\begin{array}{llll}
{[\underline{\Phi}]_{0}} & & & \\
& {[\underline{\Phi}]_{1}} & & \\
& & \ldots & \\
& & & {[\underline{\Phi}]_{N / 2}}
\end{array}\right]
$$




$$
\begin{aligned}
& {[\underline{Q}]=\left[\begin{array}{llll}
{\left[\underline{Q}_{0}\right.} & & & \\
& {[\underline{Q}]_{1}} & & \\
& & \ldots & \\
& & & {[Q]_{N / 2}}
\end{array}\right]} \\
& \left\{\underline{u_{m}}\right\}=\left(\left\{\underline{u_{m}}\right\}_{0}^{T},\left\{\underline{u_{m}}\right\}_{1}^{T},\left\{\underline{u_{m}}\right\}_{2}^{T}, \ldots,\left\{\underline{u_{m}}\right\}_{N / 2}^{T}\right)^{T} \\
& \left\{\underline{P_{m}}\right\}=\left(\left\{\underline{P_{m}}\right\}_{0}^{T},\left\{\underline{P_{m}}\right\}_{1}^{T},\left\{\underline{P_{m}}\right\}_{2}^{T}, \ldots,\left\{\underline{P_{m}}\right\}_{N / 2}^{T}\right)^{T} \\
& {[\underline{K}]=\left[\begin{array}{llll}
{[\underline{K}]_{0}} & & & \\
& {[\underline{K}]_{1}} & & \\
& & \cdots & \\
& & & {[\underline{K}]_{N / 2}}
\end{array}\right]}
\end{aligned}
$$

where

$$
\begin{gathered}
\left\{\underline{\left.u_{m}\right\}_{i i}}=[\underline{K}]_{i i}^{-1}\left\{\underline{\left.P_{m}\right\}_{i i}, i i=0,1,2, \ldots, N / 2}\right.\right. \\
{[\underline{K}]_{i i}=[\underline{\Phi}]_{i i}[\underline{Q}]_{i i}^{-1}, i i=0,1,2, \ldots, N / 2}
\end{gathered}
$$

Therefore, instead of solving Eq. (51), only a series of smaller independent system equations of Eq. (99) needs to be solved.

If eigenvalue problems of Eq. (48) and Eq. (90) are solved using the QR algorithm, the operations of multiplication and addition can be estimated by [27] and [28]

$$
\begin{aligned}
& E C_{E}=\frac{7}{3} M^{3}+4 r M^{2} \quad(\text { for Eq (48)) } \\
& E P_{E}=\frac{28 N-42}{3 N^{3}} M^{3}+\frac{8 N-8}{N^{2}} r M^{2} \quad(\text { for Eq (90)) } \\
& R_{E}=\frac{E C_{E}}{E P_{E}}=\frac{7 N^{3} M+12 N^{3} r}{(28 N-42) M+24 N^{2} r-24 N r}
\end{aligned}
$$

where $M$ denotes the dimension of matrix, $r$ denotes the number of iteration.

If inverse matrixes of $[K]$ and $[\underline{K}]_{i i}(i i=0,1,2, \ldots, N / 2)$ are generated using the Gauss elimination technique, the operations of multiplication and addition can be estimated by [27] and [28]

$$
\begin{aligned}
& E C_{K}=\frac{5}{6} M^{3}+M^{2}-\frac{5}{6} M \quad(\text { for }[K]) \\
& E P_{K}=\frac{10 N-15}{3 N^{3}} M^{3}+\frac{2 N-2}{N^{2}} M^{2}-\frac{5}{6} M \quad\left(\text { for }[\underline{K}]_{i i}\right)
\end{aligned}
$$




$$
R_{K}=\frac{E C_{K}}{E P_{K}}=\frac{N^{3}\left(5 M^{2}+6 M-5\right)}{(10 N-15) M^{2}+\left(12 N^{2}-12 N\right) M-5 N^{3}}
$$

Fig.4 and Fig.5 give a variation of $R_{E}$ and $R_{K}$ with $M$ and $N$

Actually, the real speed-up gained by partition also depends on the solver, computer used, and art of programming etc. In this paper, computing program is coded in MATLAB with its own solvers, and run on a PC computer with processor: Intel(R) Core(TM) i5-2400 CPU@3.10GHz(4 CPUs).

\section{Numerical examples}

\section{Example 1 - A hexagonal viscoelastic plate}

Consider a hexagonal viscoelastic plate subjected to uniform tension as shown in Fig. 6 where the length of hexagonal side is $2 \mathrm{~m}, P=1 \mathrm{~N} / \mathrm{m}^{2}, N=6, E_{1}=1 \times 10^{3} \mathrm{~N} / \mathrm{m}^{2}$, $v=0.25, E_{2}=2 \times 10^{3} \mathrm{~N} / \mathrm{m}^{2}, \eta=1000 \mathrm{~N} \cdot \mathrm{s} / \mathrm{m}^{2}$, plane stress conditions were assumed. The distribution of nodes on the basic region is shown in Fig.7.

Fig. 8 and Fig.9 exhibit comparisons of displacement at point A and B given by the proposed algorithm and the ABAQUS.

Tab.1 shows the comparisons of $t_{E}, t_{K}$ and $t_{T}$ with and without partitioning. $t_{E}$ is the CPU time to solve eigenvalue equation one time; $t_{K}$ is the CPU time spending on two parts : 1. generating $[K]^{-1}$ or $[\underline{K}]_{i i}^{-1}$ one time, 2. calculating all $\left\{u_{m}\right\}=[K]^{-1}\left\{P_{m}\right\}$ or $\left\{\underline{u_{m}}\right\}_{i i}=[\underline{K}]_{i i}^{-1}\left\{\underline{P_{m}}\right\}_{i i}$ for all time intervals; $t_{T}$ refers to the total CPU time consuming on the whole solution process.

Fig.10 exhibits the variation of $R_{C P}$ with time $t$, and $R_{C P}$ is defined by

$$
R_{C P}=\frac{t_{T} \text { without partitioning }}{t_{T} \text { with partitioning }}
$$

\subsection{Example 2 - A dodecagon viscoelastic plate}

Consider a dodecagon viscoelastic plate subjected to uniform tension as shown in Fig.11 where $R=1 \mathrm{~m}, P=1 \mathrm{~N} / \mathrm{m}^{2}, N=12, \quad E_{1}=1000 \mathrm{~N} / \mathrm{m}^{2}, \quad E_{2}=2000 \mathrm{~N} / \mathrm{m}^{2}$, $\eta=1000 \mathrm{~N} \cdot \mathrm{s} / \mathrm{m}^{2}, v=0.25$. The distribution of nodes on the basic region is shown in Fig.12. 
Tab.2 and Tab.3 show the comparisons of $t_{E}, t_{K}$ and $t_{T}$ with and without partitioning.

Fig.13 and Fig.14 exhibit the variation of $R_{C P}$ with $n$ and $\Delta t$, respectively.

Fig.15 exhibits the variation of the number of recursion with $t$ using different $\Delta t$. With the increase of the size of time step, more recursive computing is required to satisfy Eq. (57).

\section{Computing Remarks}

(1) A good agreement can be observed in the comparison of solutions given by the proposed algorithm and ABAQUS. By contrast with the FEM based ABAQUS, fairly less number of DOF of unknowns are used in SBFEM analysis. For instance, in the Example 1, 768 DOF is used in the proposed algorithm, while 20624 DOF is used in ABAQUS.

(2) Exploiting cyclic symmetry, a substantial speed up can be achieved in solving the eigenvalue and system equations of SBFEM, as shown in Tab.2. Since the coefficient matrices of eigenvalue and system equations of SBFEM keep invariant during the whole recursive process, the Eq. (90) needs to be solved only one time, and the inverse matrix of $[\underline{K}]_{i i}$ in Eq. (99) need to be generated only one time, the speed up gained from partitioning eigenvalue and system equations will be gradually diluted with time increase. A higher speed up can be expected when Eq. (90) and Eq. (99) are solved using parallel computing skills.

\section{Conclusions}

This paper attempts to develop a SBFEM based numerical method via a combination with a temporally adaptive algorithm for the viscoelastic analysis, and exploit the cyclic symmetry to reduce its computational expense. The proposed approach may be particularly beneficial to solve viscoelastic problems with cyclic symmetry, unbounded domains and stress singularity. The major merits include

a) In virtue of a combination of SBFEM with a temporally adaptive algorithm, a spatially and temporally coupled viscoelastic problem is converted into a series of recurrent spatial problems which are solved by SBFEM that is semi-analytical, and advantageous in dealing with the problems involving unbounded domains and singularity. A self-adaptive computation can be realized in the time domain to adapt the change of the size of time step, and results in a more precise description for the 
variation of variables.

b) For cyclically symmetric structures, the recursive coefficient matrices of eigenvalue and system equations of SBFEM are proved block-circulant, and can be partitioned into a number of smaller independent problems to solve. Thus the whole computing process is speeded up via the proposed recursive partitioning algorithm. Two numerical examples are provided to verify the proposed approach, and illustrate the advantages of proposed approach.

c) A higher computing efficiency can be expected since the proposed partitioning algorithm facilitates parallel processing.

\section{Acknowledgements}

The research leading to this paper is funded by NSF [11572068, 11202046, and 11572077], NKBRSF [2015CB057804], Natural Science Funding of Liaoning Province [2015020141] and the Fundamental Research Funds for the Central Universities [DUT14LK10, DUT14RC(4)38]. 


\section{References}

[1] R.M. Christensen, Theory of viscoelasticity: an introduction, Academic Press, 1982.

[2] E. Ahmadi, H. Barikloo, M. Kashfi, Viscoelastic finite element analysis of the dynamic behavior of apple under impact loading with regard to its different layers, Comput. Electron. Agr. 121 (2016) 1-11.

[3] L. Liu, H.T. Yang, FE analysis based on Lagrange multipliers method and group theory for structures of cyclic symmetry under arbitrary displacement boundary conditions, Chinese J. Comput. Mech. 21 (2004) 425-429.

[4] H.T. Yang, L. Liu, Z. Han, The use of cyclic symmetry in two-dimensional elastic analysis by the element-free Galerkin method, Commun. Numer. Meth. Eng. 21 (2005) $83-95$.

[5] L. Liu, H.T. Yang, A paralleled element-free Galerkin analysis for structures with cyclic symmetry, Eng. Comput. 23 (2007) 137-144.

[6] H. Ashrafia, M. Shariyata, S.M.R. Khalilia, K. Asemib, A boundary element formulation for the heterogeneous functionally graded viscoelastic structures, Appl. Math. Comput. 225 (2013) 246-262.

[7] Q.W. Xu, Prozzi JA. A time-domain finite element method for dynamic viscoelastic solution of layered-half-space responses under loading pulses, Comput. Struct. 160 (2015) 20-39.

[8] V.K. Kalyania, Pallavikab, S.K. Chakrabortyb, Finite-difference time-domain method for modelling of seismic wave propagation in viscoelastic media, Appl. Math. Comput. 237(2014) 133-145.

[9] J.P. Wolf, C. Song, Finite-element modelling of unbounded media, Chichester: John Wiley and Sons, 1996.

[10] J.P. Wolf, The scaled boundary finite element method, Chichester: John Wiley and Sons, 2003.

[11] A.J. Deeks, J.P. Wolf, A virtual work derivation of the scaled boundary finite-element method for elastostatics, Comput. Mech. 28 (2002) 489-504.

[12] G. Lin, S. Lu, J. Liu, Duality system-based derivation of the modified scaled boundary finite element method in the time domain and its application to anisotropic soil, Appl. Math. Model. 40 (2015) 5230-5255.

[13] E.T. Ooi, Z.J. Yang, Modelling crack propagation reinforced concrete using a hybrid finite element-scaled boundary finite element method, Eng. Fract. Mech. 
78(2010) 252-273.

[14] E.T. Ooi, Z.J. Yang, A hybrid finite element-scaled boundary finite element method for crack propagation modelling, Comput. Meth. Appl. Mech. Eng. 199 (2010) $1178-1192$.

[15] E.T. Ooi, Z.J. Yang, Modelling dynamic crack propagation using the scaled boundary finite element method, Int. J. Numer Meth. Eng. 88 (2011) 329-349. [16] Z.J. Yang, A.J. Deeks, H. Hao, Transient dynamic fracture analysis using scaled boundary finite element method: a frequency domain approach, Eng. Fract. Mech. 74 (2007) 669-687.

[17] M.H. Bazyar, A. Talebi, Scaled boundary finite-element method for solving non-homogeneous anisotropic heat conduction problems, Appl. Math. Model. 39 (2015) $7583-7599$.

[18] S. Hell, W. Becker, The scaled boundary finite element method for the analysis of 3D crack interaction, J. Comput. Sci. 9 (2015) 76-81.

[19] H.T. Yang, A precise algorithm in time domain to solve the problem of heat transfer, Num. Heat. Tr B-Fund. 35 (1999) 243-249.

[20] Z. Han, H.T. Yang, L. Liu, Solving viscoelastic problems with cyclic symmetry via a precise algorithm and EFGM, Acta. Mech. Sinica. 22 (2006) 170-176.

[21] H.T. Yang, A new approach of time stepping for solving transfer problems. Commun. Numer. Meth. Eng. 15 (1999) 325-334.

[22] Y.Q. He, H.T. Yang, M. Xu, A scaled boundary finite element method for cyclically symmetric two-dimensional elastic analysis, Comput. Struct. 120 (2013) 1-8.

[23] Y.Q. He, H. T Yang, Solving viscoelastic problems by combining SBFEM and a temporally piecewise adaptive algorithm, submitted to Mechanics of Time-Dependent.

[24] O.C. Zienkiewicz, K. Morgan, Finite Element and Approximation, Wiley-Interscience Publication, 1983.

[25] I.H. Shames, F.A. Cozzarelli, Elastic and inelastic stress analysis, Prentice Hall, 1992.

[26] S.R. Chidgzey, J. Trevelyan, A.J. Deeks, Coupling of the boundary element method and the scaled boundary finite element method for computations in fracture mechanics, Comput. Struct. 86 (2008) 1198-1203.

[27] S.F. Xu, The theory and method of matrix computation, Peking University Press, China, 2001.

[28] L.J. Ding, Numerical Methods, Beijing Institute of Technology Press, China, 2005. 


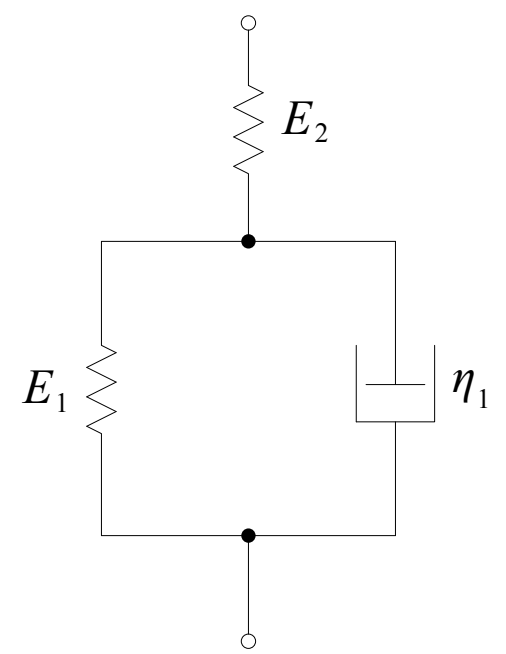

Fig.1 Three-parameter solid viscoelastic model 


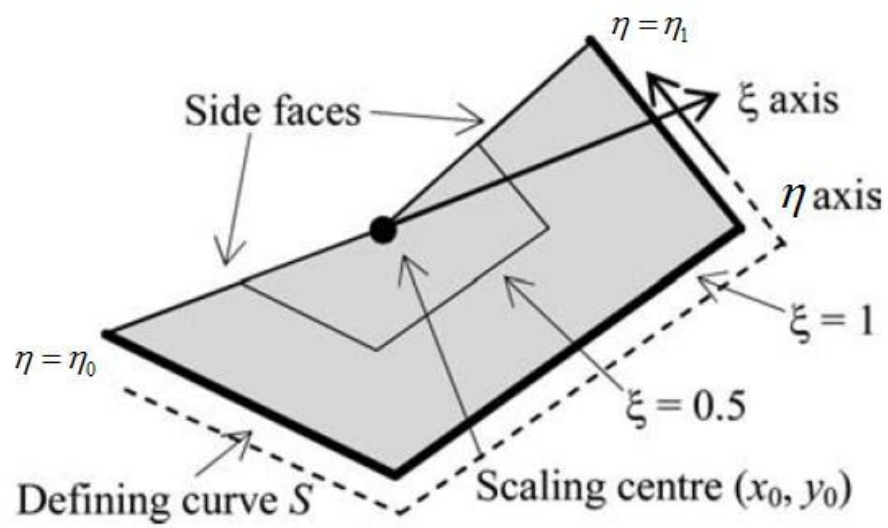

Fig.2 Scaled boundary coordinate system 


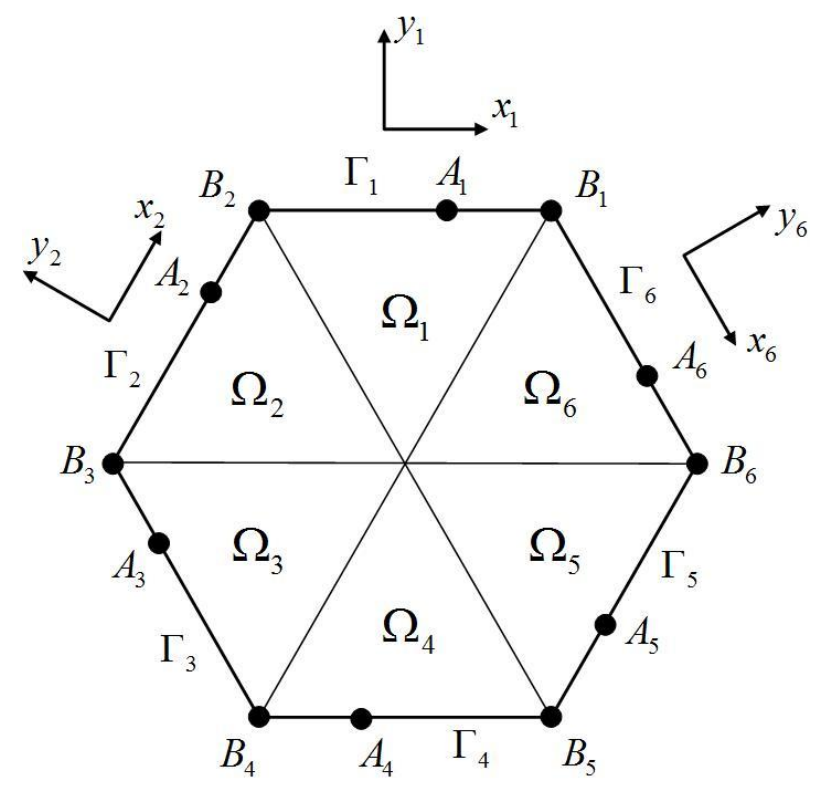

Fig.3 A rotationally periodic symmetric plane with $N=6$ 


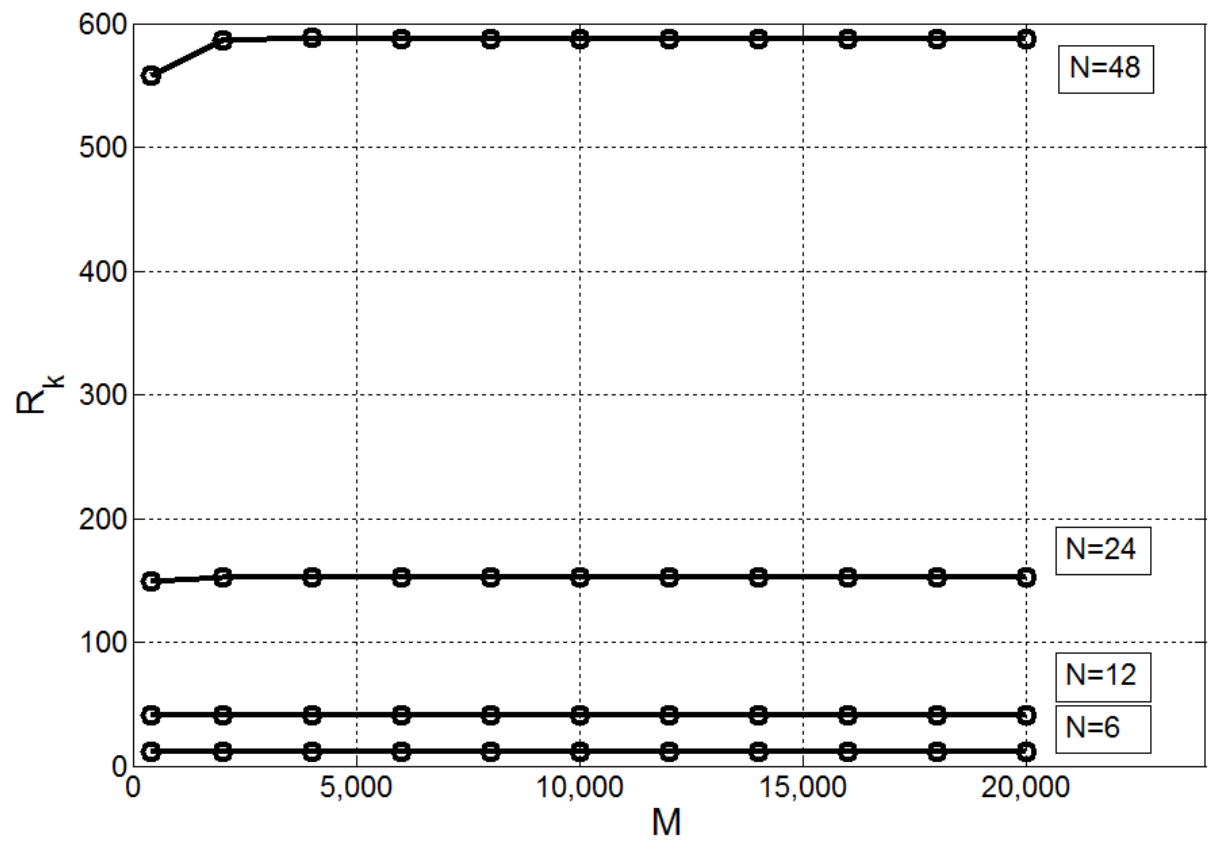

Fig.4.The variation of $R_{K}$ with $M$ and $N$ 


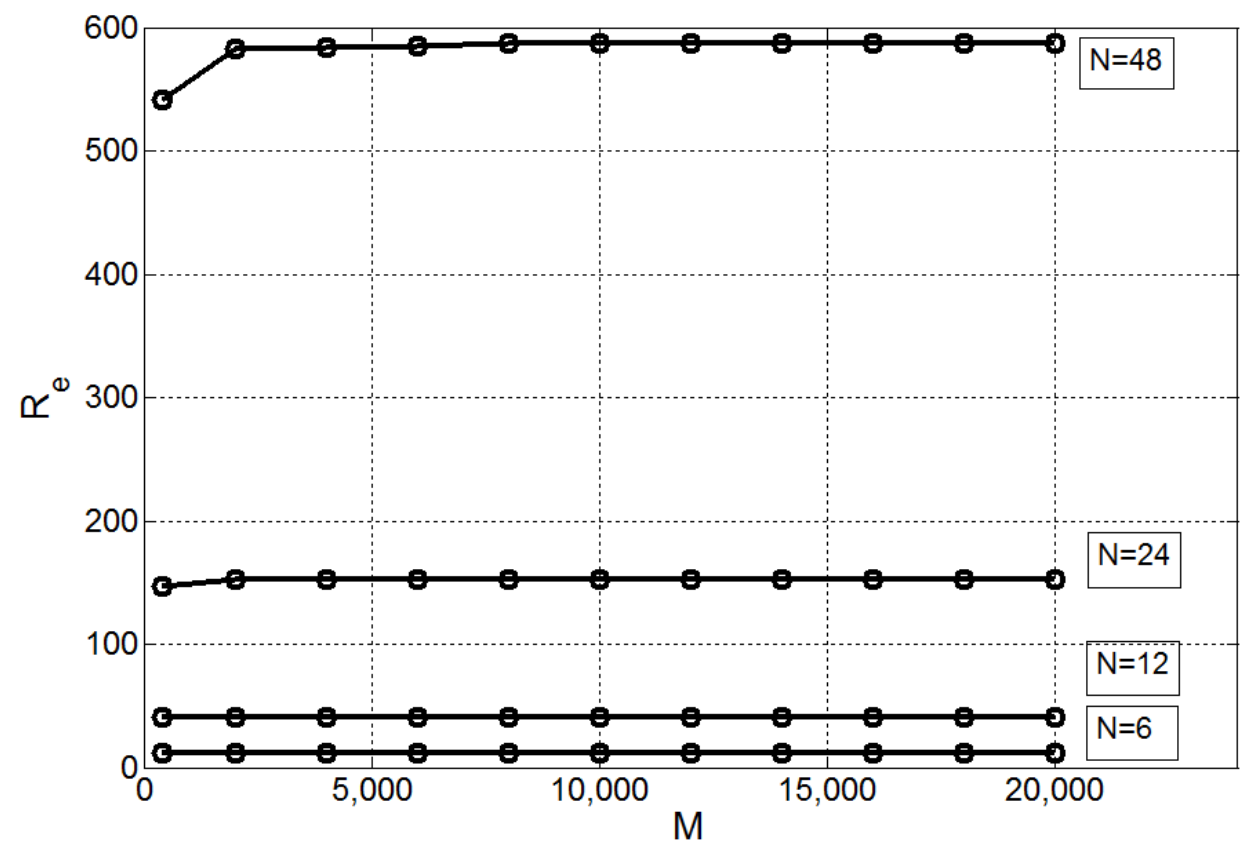

Fig.5.The variation of $R_{E}$ with $M$ and $N \quad(r=1)$ 


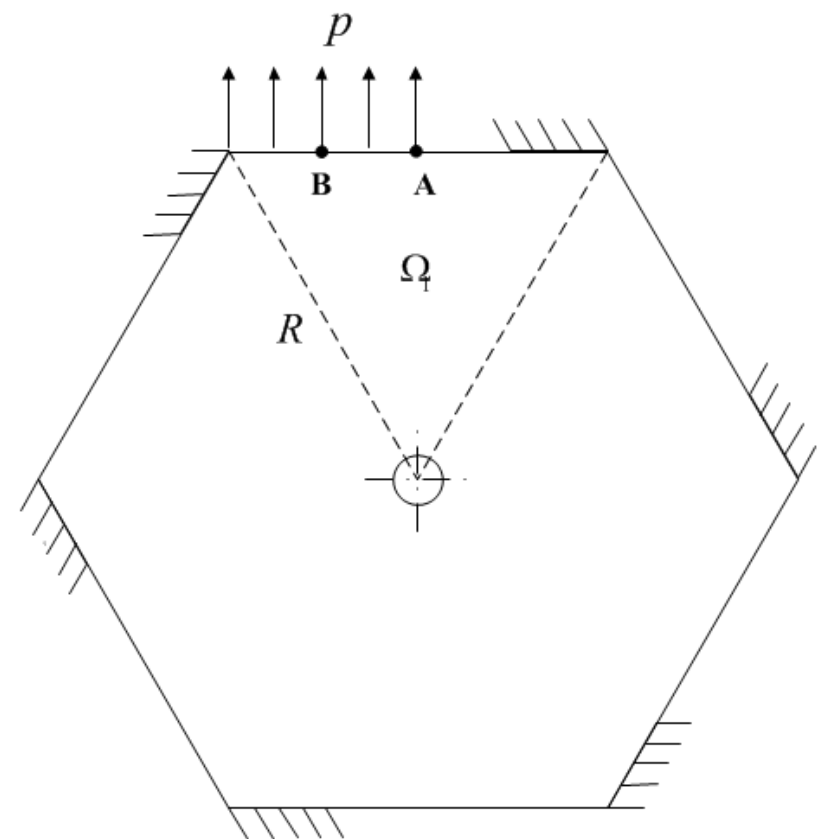

Fig.6.A hexagonal plate 


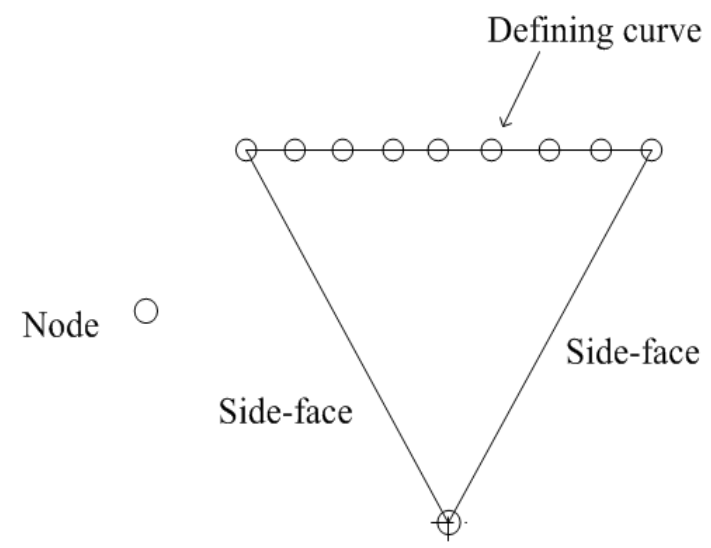

Scaling centre

Fig.7. Nodes distribution on the basic region 


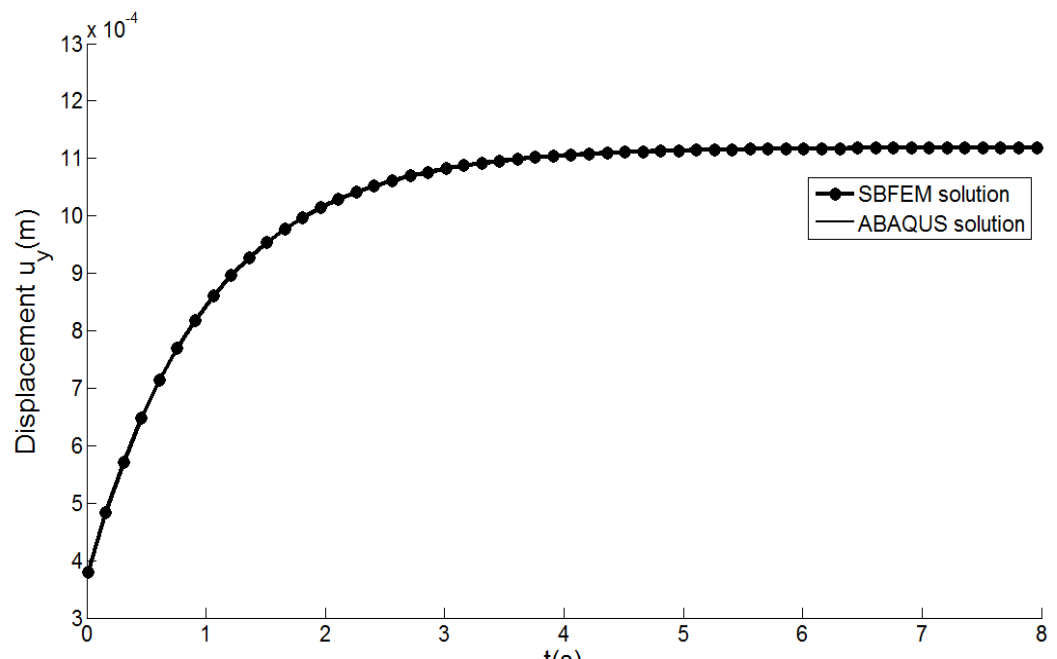

$t(s)$

Fig.8. A comparison of $u_{y}$ with $t$ at point A $(0, \sqrt{3})$ 


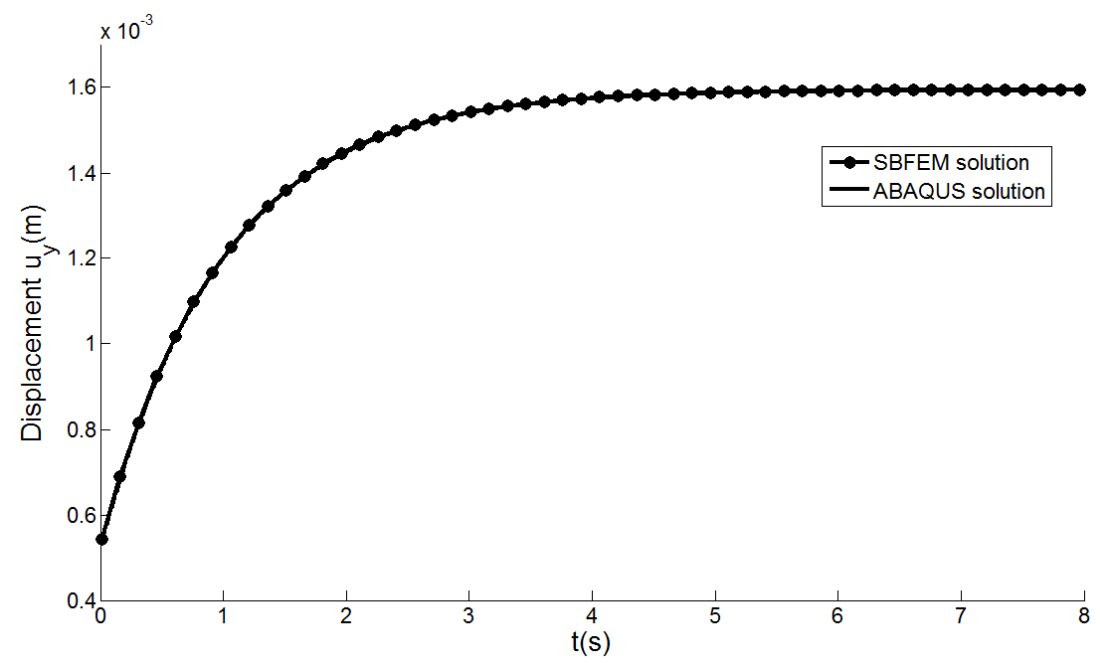

Fig.9. A comparison of $u_{y}$ with $t$ at point B $(-0.5, \sqrt{3})$ 


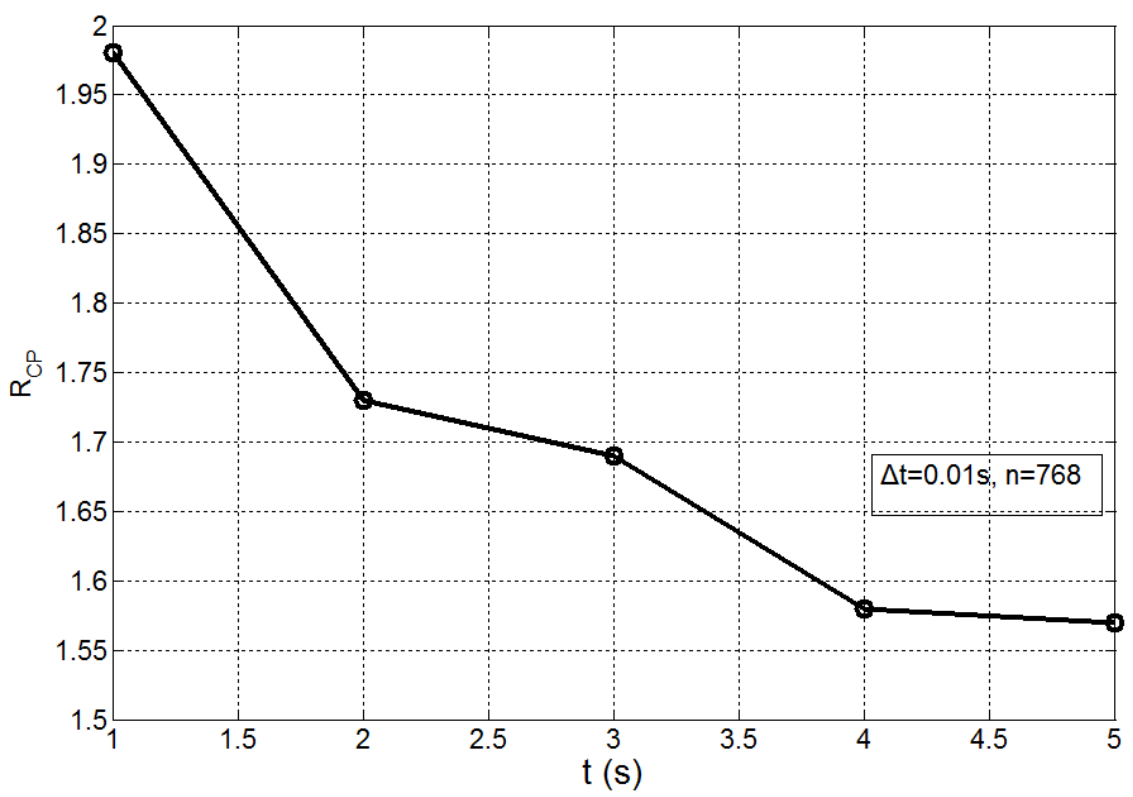

Fig.10.The variation of $R_{C P}$ with $t$ 


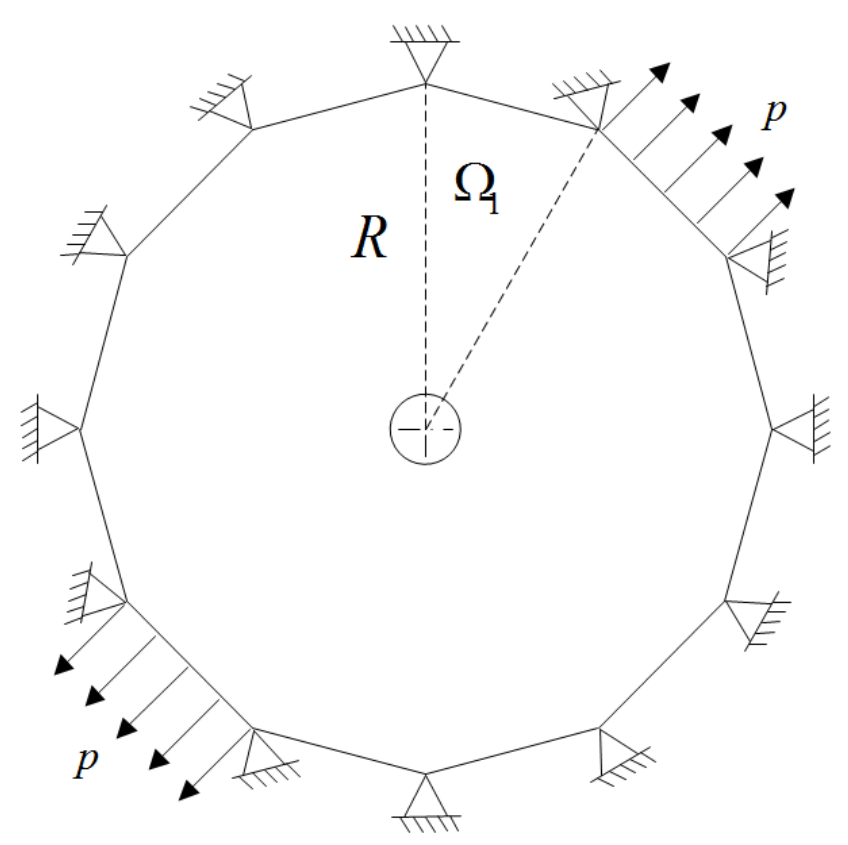

Fig.11.A dodecagon plate 


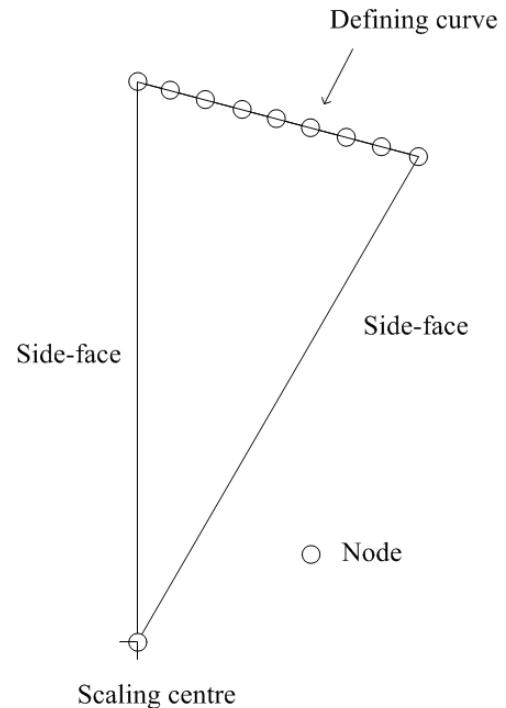

Fig.12. Nodes distribution on the basic region 


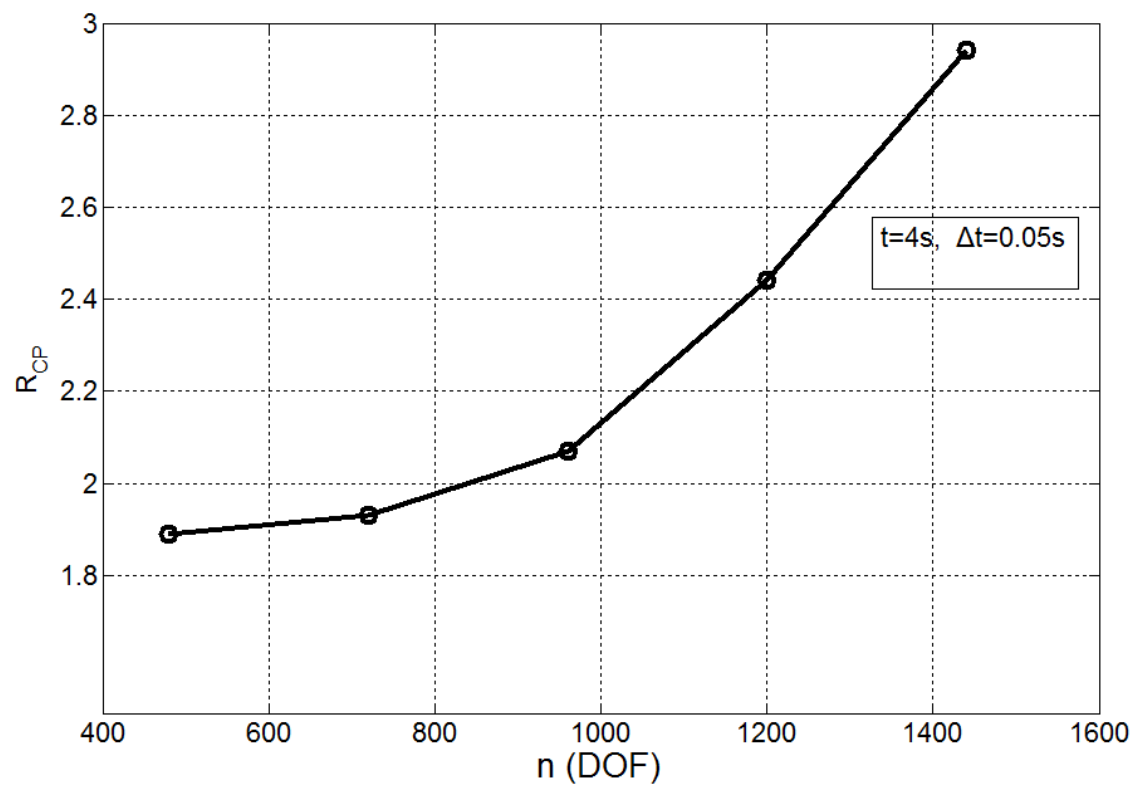

Fig.13.The variation of $R_{C P}$ with $n$ 


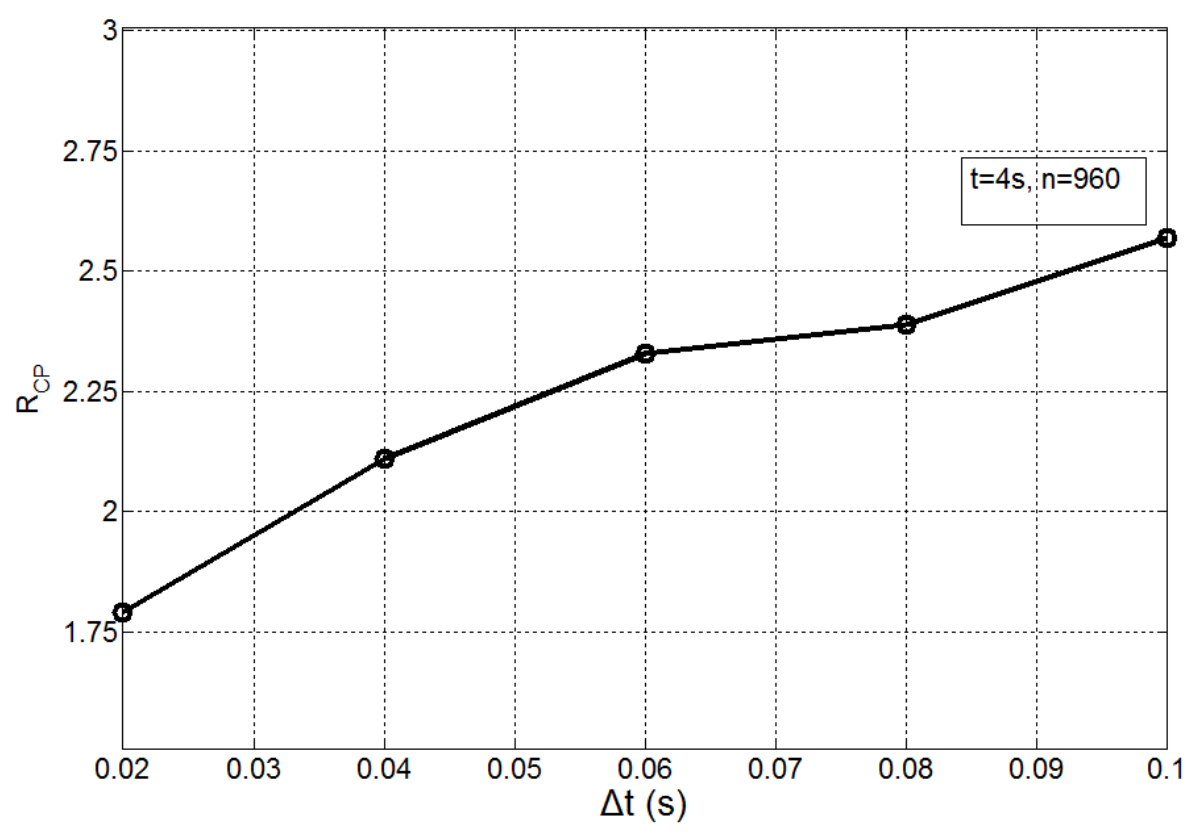

Fig.14.The variation of $R_{C P}$ with $\Delta t$ 


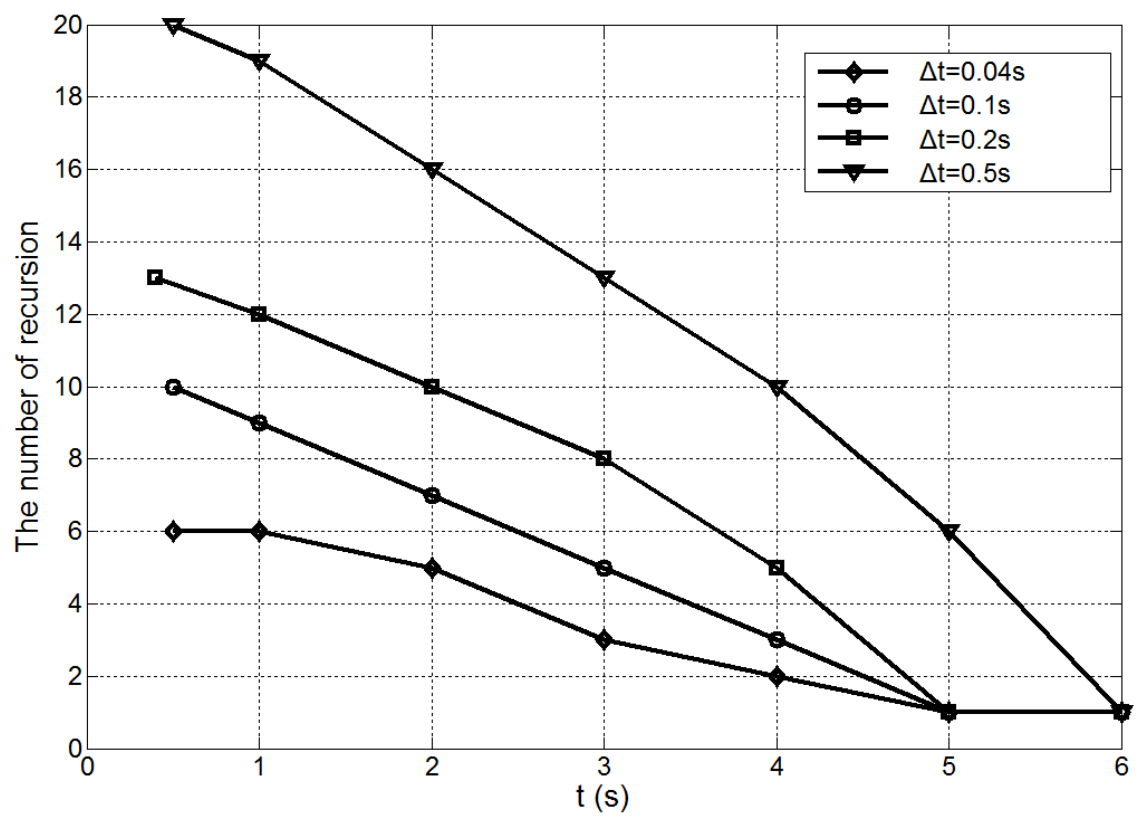

Fig.15.The variation of the number of recursion with $\Delta t$ and $t \quad\left(\beta=1 \times 10^{-10}\right)$ 
Table 1 Comparison of CPU time $(\Delta t=0.01 \mathrm{~s}, n=768)$

\begin{tabular}{|c|c|c|c|c|}
\hline \multicolumn{1}{|c|}{} & $\begin{array}{c}\text { SBFEM without } \\
\text { partitioning } \\
\text { Time } \mathrm{t}_{1}(\mathrm{~s})\end{array}$ & $\begin{array}{c}\text { SBFEM with } \\
\text { partitioning } \\
\text { Time } \mathrm{t}_{2}(\mathrm{~s})\end{array}$ & Ratio $\mathrm{t}_{1} / \mathrm{t}_{2}$ \\
\hline \multirow{2}{*}{$t=1 \mathrm{~s}$} & 18.52 & 3.32 & 5.58 \\
\cline { 2 - 5 } & $t_{E}$ & 12.62 & 8.78 & 1.44 \\
\hline \multirow{3}{*}{$t=3 \mathrm{~s}$} & $t_{K}$ & 36.21 & 18.27 & 1.98 \\
\cline { 2 - 5 } & $t_{K}$ & 36.43 & 26.01 & 1.40 \\
\hline \multirow{2}{*}{$t=5 \mathrm{~s}$} & $t_{T}$ & 59.92 & 35.49 & 1.69 \\
\cline { 2 - 5 } & $t_{K}$ & 56.33 & 41.87 & 1.57 \\
\hline
\end{tabular}


Table 2 Comparison of CPU time $(t=4 \mathrm{~s}, \Delta t=0.05 \mathrm{~s})$

\begin{tabular}{|c|c|c|c|c|}
\hline & & $\begin{array}{l}\text { SBFEM without } \\
\text { partitioning } \\
\text { Time } t_{1}(s)\end{array}$ & $\begin{array}{l}\text { SBFEM with } \\
\text { partitioning } \\
\text { Time } t_{2}(s)\end{array}$ & Ratio $t_{1} / t_{2}$ \\
\hline \multirow{3}{*}{$n=480$} & $t_{E}$ & 4.74 & 0.55 & 8.62 \\
\hline & $t_{K}$ & 4.52 & 2.92 & 1.55 \\
\hline & $t_{T}$ & 13.28 & 7.04 & 1.89 \\
\hline \multirow{3}{*}{$n=960$} & $t_{E}$ & 33.16 & 2.14 & 15.50 \\
\hline & $t_{K}$ & 22.34 & 20.17 & 1.11 \\
\hline & $t_{T}$ & 64.69 & 31.25 & 2.07 \\
\hline \multirow{3}{*}{$n=1440$} & $t_{E}$ & 116.02 & 5.43 & 21.37 \\
\hline & $t_{K}$ & 44.59 & 39.11 & 1.14 \\
\hline & $t_{T}$ & 185.78 & 63.26 & 2.94 \\
\hline
\end{tabular}


Table 3 Comparison of CPU time $(n=960, t=4 \mathrm{~s})$

\begin{tabular}{|c|c|c|c|c|}
\hline \multicolumn{1}{|c|}{} & $\begin{array}{c}\text { SBFEM without } \\
\text { partitioning } \\
\text { Time } \mathrm{t}_{1}(\mathrm{~s})\end{array}$ & $\begin{array}{c}\text { SBFEM with } \\
\text { partitioning } \\
\text { Time } \mathrm{t}_{2}(\mathrm{~s})\end{array}$ & Ratio $\mathrm{t}_{1} / \mathrm{t}_{2}$ \\
\hline \multicolumn{2}{|c|}{$t_{E}$} & 32.50 & 2.04 & 15.93 \\
\hline \multirow{3}{*}{$\Delta t=0.02 \mathrm{~s}$} & $t_{K}$ & 44.29 & 38.06 & 1.16 \\
\cline { 2 - 6 }$\Delta t=0.06 \mathrm{~s}$ & $t_{T}$ & 87.39 & 48.78 & 1.79 \\
\cline { 2 - 6 } & $t_{K}$ & 17.64 & 15.55 & 1.13 \\
\hline \multirow{3}{*}{$\Delta t=0.1 \mathrm{~s}$} & $t_{T}$ & 61.02 & 26.22 & 2.33 \\
\cline { 2 - 6 } & $t_{K}$ & 12.96 & 10.75 & 1.21 \\
\hline
\end{tabular}

